\section{ORIGINAL RESEARCH}

\section{R.P.H. Bokkers}

F.J. Wessels

H.B. van der Worp

J.J.M. Zwanenburg

W.P.Th.M. Mali

J. Hendrikse

\title{
Vasodilatory Capacity of the Cerebral Vasculature in Patients with Carotid Artery Stenosis
}

BACKGROUND AND PURPOSE: Impairment of the cerebral autoregulation is an important predictor of TIA and stroke in patients with an ICA stenosis. The autoregulative status can be assessed directly by measuring the vasodilatory capacity of the cerebral arteries. The aim of our study was to investigate the vasodilatory capacity of the proximal and distal cerebral vasculature in patients with an ICA stenosis and healthy control subjects by combining MRA with an acetazolamide provocation challenge.

MATERIALS AND METHODS: Fourteen functionally independent patients (mean age, $67.2 \pm 8.7$ years) with a symptomatic ICA stenosis and 19 healthy controls (mean age, $63.1 \pm 7.2$ years) were included. MRA was performed before and 20 minutes after intravenous administration of acetazolamide. The vasodilatory capacity of 11 proximal and distal cerebral vessels was assessed by measuring the increase in vessel diameter after acetazolamide.

RESULTS: In the hemisphere ipsilateral to the ICA stenosis, there was no increase in diameter after acetazolamide, whereas a significant increase was measured in the contralateral hemisphere for the A1 and A2 segments of the ACA, the pericallosal artery, and the BA. A significant diameter increase was measured in all except 1 vessel of the controls. The vasodilatory capacity was significantly lower ipsilateral to the ICA stenosis compared with the A1 segment of the ACA and the P2 segment of the PCA in the controls.

CONCLUSIONS: MRA combined with an acetazolamide provocation challenge can measure normal and impaired vasodilatory capacity of the cerebral vasculature.

ABBREVIATIONS: $\mathrm{ACA}=$ anterior cerebral artery; $\mathrm{AcomA}=$ anterior communicating artery; $\mathrm{BA}=$ basilar artery; $\mathrm{CO}_{2}=$ carbon dioxide; FWHM = full width at half maximum; ICA = internal carotid artery; MRA = MR angiography; PCA = posterior cerebral artery; PcomA = posterior communicating artery; TIA = transient ischemic attack; TOF = time of flight

mpairment of the cerebral autoregulation is an important predictor of TIAs and stroke in patients with an ICA stenosis. ${ }^{1-4}$ The cerebral autoregulation enables the brain to keep cerebral blood flow constant during fluctuations of the arterial blood pressure. ${ }^{5}$ A steno-occlusive lesion in $\geq 1$ of the feeding arteries of the brain leads to a reduction of the perfusion pressure in the vasculature distal to the lesion. ${ }^{6}$ As perfusion pressure decreases, cerebral blood flow is initially maintained by recruiting collateral blood flow through the circle of Willis, leptomeningeal arteries, or ophthalmic artery. ${ }^{7,8}$ When collateral blood flow falls short, the cerebral arteries and arterioles dilate to reduce the vascular resistance to arterial inflow. ${ }^{9}$ With a further reduction of cerebral perfusion pressure, the autoregulative capacity of the brain is overcome and cerebral blood flow decreases. ${ }^{8}$

A variety of different imaging methods has been developed for assessing the cerebral autoregulative status. Most methods measure the presence and degree of dilation of the cerebral vasculature before and after a vasodilatory stimulus by exam-

Received May 24, 2010; accepted after revision October 25

From the Department of Radiology (R.P.H.B., W.P.Th.M.M., J.H.) and Image Sciences Institute (J.J.M.Z.), University Medical Center Utrecht, Utrecht, the Netherlands; Department of Radiology (F.J.W.), Meander Medical Center, Amersfoort, the Netherlands; and Department of Neurology (H.B.v.d.W.), Rudolf Magnus Institute of Neuroscience, University Medical Center Utrecht, Utrecht, the Netherlands.

Please address correspondence to R.P.H. Bokkers, MD, Department of Radiology, University Medical Center Utrecht, Heidelberglaan 100, HP E.01.132, 3584 CX Utrecht, the Netherlands; e-mail: r.p.h.bokkers@umcutrecht.nl

Indicates article with supplemental on-line tables.

DOI 10.3174/ajnr.A2438 ining the flow velocity of blood through the major cerebral arteries or with quantitative measurements of cerebral blood flow at the brain-tissue level. ${ }^{7,10,11}$ With sonography and traditional conventional angiography, studies have been able to directly assess the autoregulative capacity of the brain vasculature, by measuring the vasodilatory capacity of the cerebral arteries. However, these studies have primarily focused on the proximal cerebral arteries. ${ }^{12-15}$ Recent advances in MRA have enabled noninvasive visualization of cerebral arteries with increased detail. With 3T MRA, it is possible to directly and reliably measure changes in the diameter of intracranial vessels. ${ }^{16}$

The aim of our study was to investigate the vasodilatory capacity of the proximal and distal cerebral vasculature in patients with an ICA stenosis and healthy control subjects by combining MRA with an acetazolamide-provocation challenge.

\section{Materials and Methods}

The study was approved by the institutional review board, and written informed consent was obtained from all participants.

\section{Subjects}

Fourteen patients ( 8 men and 6 women; mean age, $67.2 \pm 8.7$ years) with a symptomatic ICA stenosis of $>50 \%$ referred consecutively to the department of radiology for diagnosis and grading of the ICA stenosis and 19 healthy volunteers (6 men and 13 women; mean age, $63.1 \pm 7.2$ years) were included in the study. All patients had experienced a TIA or nondisabling ischemic stroke on the side of the ICA stenosis. Patients who had diabetes mellitus or severe renal or liver 

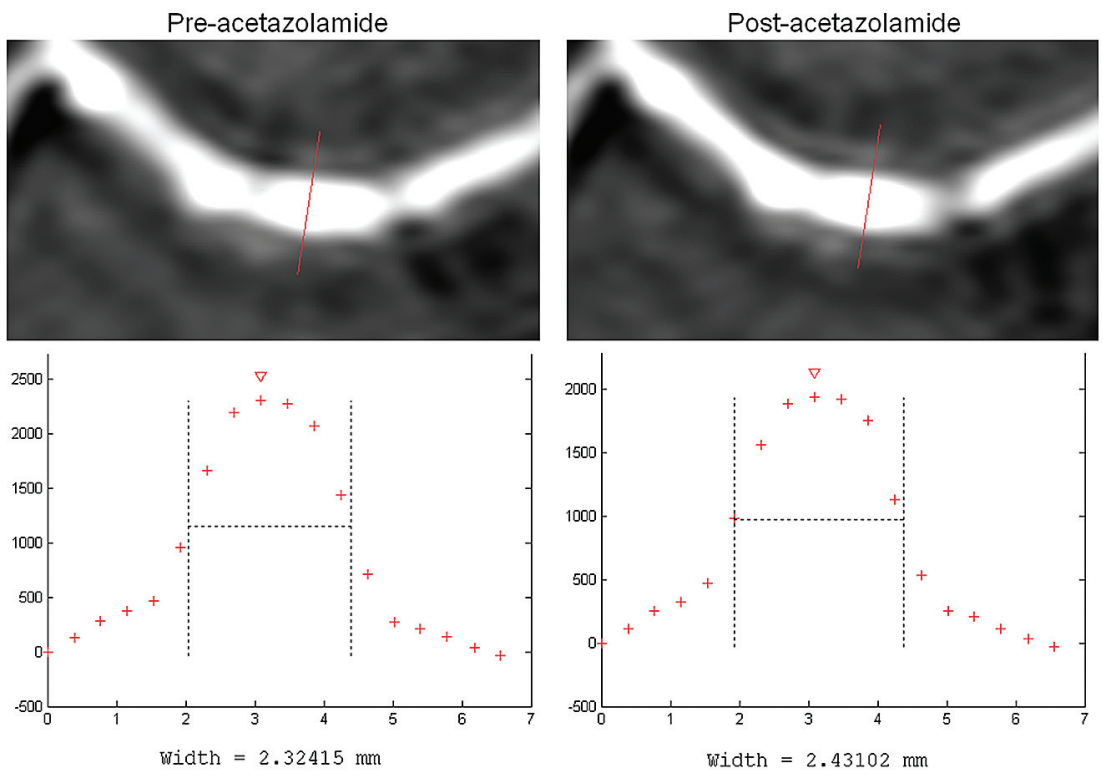

Fig 1. Diameter measurement of the M1 segment of the MCA in a healthy subject by using the FWHM method.

dysfunction or who had experienced a severe stroke causing major disability (modified Rankin Scale score of 3-5) were excluded from the study. ${ }^{17}$ Diagnosis and grading of the ICA stenosis was performed with duplex ultrasonography. Healthy volunteers were recruited through local media advertisements. All were without a history of neurologic disease, vascular pathology on MR imaging or MRA of the brain, or a stenosis of the ICA.

\section{MR Imaging}

The MR imaging investigations were performed on a 3T scanner (Achieva; Philips Healthcare, Best, the Netherlands) with a quadrature head coil for signal-intensity reception. The imaging protocol consisted of a $2 \mathrm{D}$ phase-contrast sagittal localizer survey through the circle of Willis, followed by a 3D-TOF MRA sequence with the following parameters: TR, $23 \mathrm{~ms}$; TE, $3.5 \mathrm{~ms}$; flip angle, $18^{\circ}$; sensitivity encoding factor, 2 ; FOV, $200 \times 200 \times 100 \mathrm{~mm}^{3}$; matrix, $304 \times 200$ with 100 sections; reconstructed voxel size, $0.39 \times 0.39 \times 1.00 \mathrm{~mm}$; and acquisition time, 2 minutes 57 seconds. MRA was performed before and 20 minutes after intravenous administration of $14 \mathrm{mg} / \mathrm{kg}$ of acetazolamide with a maximum dose of $1200 \mathrm{mg}$.

\section{Data Analysis}

Measurements of the vessel diameters pre- and postacetazolamide were performed on the transversal source sections of the 3D-TOF MRA dataset. The diameter was defined as the FWHM of the vessel, calculated from an intensity profile through the center of the vessel (Fig 1). The vessel-intensity profiles were analyzed with mathematic software (Matlab, Version 6.5; MathWorks, Natick, Massachusetts) to measure the FWHM. A vessel-diameter quantification method based on FWHM was used because this provides a reproducible and precise calculation of the vessel diameter, independent of window leveling and the evaluation of vessel edges by an observer. ${ }^{18}$ We measured the diameters of the following arteries in both the ipsilateral and contralateral hemispheres: the A1 and A2 segments of the ACA; the pericallosal artery; the callosomarginal artery; the M1, M2, and M3 segments of the MCA; the P1 and P2 segments of the PCA; the PcomA; and the BA. No vessel diameter could be measured for the
AcomA with the FWHM method because of the small length and the close proximity of the ACAs.

The vasodilatory capacity was defined as the percentage of increase in arterial diameter after administration of acetazolamide. The Statistical Package for the Social Sciences for Windows, Version 15.0.1 (SPSS, Chicago, Illinois) was used for statistical analysis. Descriptive statistical analyses were performed to summarize patient characteristics. Differences between the vessel diameter before and after administration of acetazolamide were tested with a paired $t$ test. Differences in vasodilatory capacity between the hemispheres and between patients and control subjects were tested with an independent $t$ test. A $P$ value $<.05$ was considered to indicate a statistically significant difference. Values are expressed as mean \pm standard error of the mean unless otherwise specified.

\section{Results}

The baseline characteristics of the patient and control groups are given in On-line Table 1. Figure 1 shows a representative example of the vessel diameter before and after administration of acetazolamide. On-line Table 2 summarizes the vessel diameters before and after administration of acetazolamide in the hemispheres ipsilateral and contralateral to the symptomatic ICA stenosis and in the healthy control group. In the hemisphere ipsilateral to the ICA stenosis, no significant changes in the diameter of any artery were measured after administration of acetazolamide. In the hemisphere contralateral to the stenosis, a significant increase in arterial diameter was measured in the A1 segment of the ACA (1.91 \pm 0.34 versus $2.02 \pm 0.35 \mathrm{~mm}, P<.01$ ), the A2 segment of the ACA $(1.62 \pm 0.15$ versus $1.71 \pm 0.19 \mathrm{~mm}, P<.01)$, the pericallosal artery $(1.41 \pm 0.10$ versus $1.48 \pm 0.10 \mathrm{~mm}, P<.01)$, and the BA $(2.43 \pm 0.48$ versus $2.57 \pm 0.50 \mathrm{~mm}, P<.01)$. In the healthy control subjects, there were no differences in vasodilatory capacity between the left and right hemispheres; for this reason, both hemispheres were averaged for further analysis. A significant increase in vessel diameter was measured after ad- 


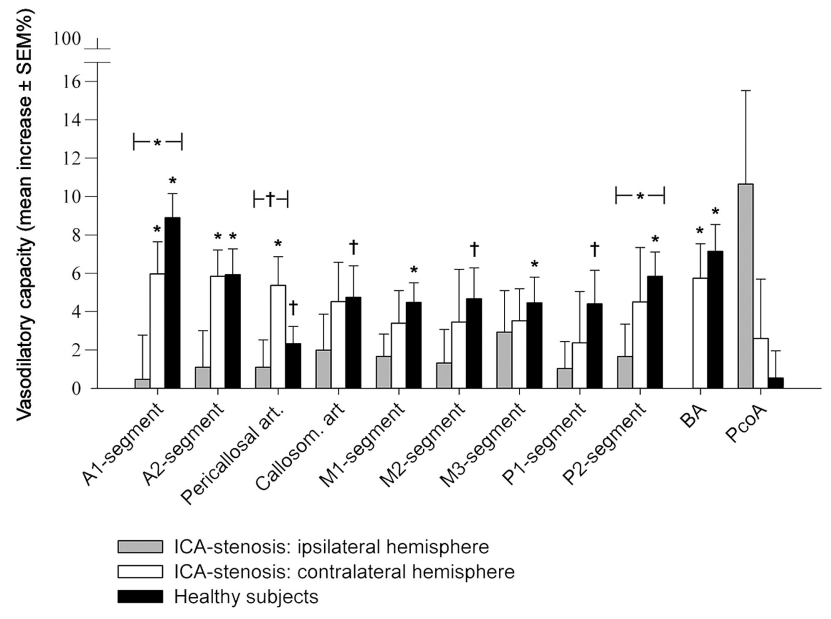

Fig 2. Diameter change of cerebral arteries in terms of percentage after administration of acetazolamide. Asterisk indicates a statistically significant $(P<.01)$ difference between the vessel diameter in the pre- and postacetazolamide scans. The brackets indicate a significant difference (asterisk indicates $P<.01$; dagger, $P<.05$ ) in vasodilatory capacity between the indicated groups.

ministration of acetazolamide in all vessels of the control subjects, with the exception of the PcomA $(P=.83)$.

Figure 2 shows the vasodilatory capacity (percentage of diameter increase) in the patients with an ICA stenosis and the healthy volunteers. The vasodilatory capacity was significantly lower in the hemisphere ipsilateral to the ICA stenosis compared with healthy volunteers, in the A1 segment of the ACA $(0.47 \pm 2.30 \%$ versus $8.90 \pm 1.26 \%, P<.01)$ and the $\mathrm{P} 2$ segment of the PCA $(1.66 \pm 1.69 \%$ versus $5.84 \% \pm 1.27 \%$, $P=.048)$. For the pericallosal artery, vasodilatory capacity was significantly lower in the ipsilateral hemisphere compared with the contralateral hemisphere $(1.10 \pm 1.43 \%$ versus $5.37 \pm 1.49 \%, P=.049)$. There were no differences in vasodilatory capacity between the contralateral hemisphere in the patients with an ICA stenosis and the healthy control subjects.

\section{Discussion}

In the present study, we show the ability of MRA to assess the vasodilatory capacity of the cerebral arteries on an acetazolamide challenge. Impaired vasodilatory capacity was found in patients with a symptomatic stenosis of the carotid artery. In the hemisphere ipsilateral to the symptomatic ICA stenosis, there were no significant increases in vessel diameter, whereas vessel diameter increased significantly in the proximal vasculature of the hemisphere contralateral to the ICA stenosis.

To our knowledge, this is the first study to show the vasodilatory effect of acetazolamide with MRA on the diameter of intracranial vasculature in patients with a carotid artery stenosis. Schoonman et $\mathrm{al}^{16}$ used a similar TOF MRA technique to investigate whether migraine headaches are associated with cerebrovascular vasodilation by provoking an attack with nitroglycerine, demonstrating that there were no changes in cerebral or meningeal blood vessel diameters during an attack. In another study performed at 1.5T, TOF MRA showed that in patients with an ICA stenosis, the diameter of the vessel in the circle of Willis is associated with the presence of collateral flow. ${ }^{19}$ TOF MRA at a field strength of $3 \mathrm{~T}$ allows a higher signal intensity-to-noise ratio on the high-resolution MRA source images, enabling visualization of smaller cerebral arteries.

The studies that have directly investigated the vasodilatory capacity of the cerebral arteries have focused on the major proximal cerebral arteries. Valdueza et $\mathrm{al}^{14}$ showed, in 16 healthy subjects, with transcranial Doppler, an increase of $9.5 \pm 7.0 \%$ in the diameter of the MCA during $\mathrm{CO}_{2}$-induced hypercapnia. Conflicting results were observed in the study of Schreiber et al, ${ }^{13}$ in which no changes in MCA diameters contralateral to a carotid artery occlusion were found in 8 patients after administration of acetazolamide. However, this study was limited by the available MR imaging spatial resolution, with which changes smaller than $10 \%$ could not reliably be detected. Using traditional angiography, Huber and Handa ${ }^{15}$ investigated vasodilation during stages of hypercapnia in both the proximal and distal vasculature, reporting vasodilation $\leq 22.5 \%$ in the smallest arteries $(0.5-1.0 \mathrm{~mm})$.

Regulation of cerebral blood flow is possible through the vasculature response of the brain to changes in the arterial blood pressure or intracranial pressure. Through myogenic (stress sensing mechanism) and neurogenic (sympathetic innervation) coupling, changes in the arterial and intracranial pressure lead to regulation of the tone of the vascular smooth muscle cells in the cerebral arteries, ranging from the large conductance arteries to the arterioles. Acetazolamide is a reversible inhibitor of the enzyme carbonic anhydrase, and though the exact mechanism is unknown, the vasodilatory effect on the vasculature of the brain is ascribed to carbonic acidosis. ${ }^{20,21}$ We show that the vasodilatory effect of acetazolamide can be assessed by measuring changes in arterial diameter throughout the vasculature of the brain. Other challenges used to measure the presence or degree of hemodynamic impairment rely predominantly on stimulating $\mathrm{CO}_{2}$-reactive arterioles by increasing the $\mathrm{CO}_{2}$ concentration through inhalation, breath-holding, or hyperventilation. The autoregulatory vasodilatory capacity can then be indirectly examined by measuring the increase in flow velocity of blood through the major cerebral arteries or with quantitative measurements of cerebral blood flow at the brain-tissue level. ${ }^{22}$

In our study, increases in vessel diameter of both the large conductory arteries and distal cerebral vasculature ranged from $1 \%$ to $9 \%$ in the healthy volunteers. Furthermore, we found an impaired vasodilatory capacity in the hemisphere ipsilateral to the symptomatic ICA stenosis. Although this was a cross-sectional analysis of patients and our results do not pertain to identifying impaired vasodilatory capacity in individuals, our findings of impaired vasodilatory capacity measured with MRA are in agreement with previous studies, which have demonstrated decreased cerebrovascular reactivity with flow measurements of the MCA and quantitative measurements of cerebral blood flow. ${ }^{6,8,23-27}$ There was a significant difference between the ipsilateral hemisphere and the healthy control group. There was only a difference in vasodilatory capacity between the ipsilateral and contralateral hemisphere within the patients with an ICA stenosis in 1 vessel, the pericallosal artery. We believe that this lack of difference may have been caused by more generalized atherosclerotic disease, which also results in a reduction in vasodilatory capacity of the vasculature on the contralateral side of a symptomatic carotid stenosis. 
A limitation of the current study is the use of TOF MRA, in which the increase in inflow signal intensity with an acetazolamide challenge may also have an effect on the signal intensity of the vasculature. Still, we think that the FWHM method will render measurements less sensitive for a potential change in signal intensity. Previously, Hoogeveen et $\mathrm{al}^{18}$ showed that the FWHM method could detect small changes in diameter, even in relatively small vessels compared with the scanning resolution of the TOF MRA images. Furthermore, we believe that a potential hemodynamic contribution to the TOF MRA diameter measurements will still make this a useful measurement to assess cerebral vasodilatory capacity. The 3D TOF MRA sequence used to measure the diameters of the intracerebral vessels before and after acetazolamide was not cardiac-gated. Pulsatile flow could, therefore, have caused signal-intensity loss and ghosting artifacts in the phase-encoding direction. Although this might have influenced the diameter measurements, this effect would be present in the measurements both before and after acetazolamide administration. The influence of cardiac pulsations on the vasodilatory-effect measurements is, therefore, expected to be limited. Analysis of the AcomA diameter in the transversal plane with the FWHM proved to be difficult due to the A2 segments lying adjacent to each other; therefore, no diameter evaluation of the AcomA could be performed in the present study.

\section{Conclusions}

This study demonstrates that acetazolamide has a direct vasodilatory effect on large intracranial arteries. MRA combined with an acetazolamide provocation challenge can measure this effect, and this vasodilatory effect is diminished in patients with a stenosis in the ICA. This may contribute, in the future, to a better understanding of the vasodilatory capacity of the cerebral vasculature and impairment in patients with cerebrovascular disease.

\section{References}

1. Markus $\mathrm{H}$, Cullinane $\mathrm{M}$. Severely impaired cerebrovascular reactivity predicts stroke and TIA risk in patients with carotid artery stenosis and occlusion. Brain 2001;124:457-67

2. Blaser T, Hofmann K, Buerger T, et al. Risk of stroke, transient ischemic attack, and vessel occlusion before endarterectomy in patients with symptomatic severe carotid stenosis. Stroke 2002;33:1057-62

3. Silvestrini M, Vernieri F, Pasqualetti P, et al. Impaired cerebral vasoreactivity and risk of stroke in patients with asymptomatic carotid artery stenosis. JAMA 2000;283:2122-27

4. Yonas H, Smith HA, Durham SR, et al. Increased stroke risk predicted by compromised cerebral blood flow reactivity. J Neurosurg 1993;79:483-89

5. Paulson OB, Strandgaard S, Edvinsson L. Cerebral autoregulation. Cerebrovasc Brain Metab Rev 1990;2:161-92

6. Powers WJ, Press GA, Grubb RL Jr. The effect of hemodynamically significant carotid artery disease on the hemodynamic status of the cerebral circulation. Ann Intern Med 1987;106:27-35

7. Derdeyn CP, Grubb RL, Powers WJ. Cerebral hemodynamic impairment: methods of measurement and association with stroke risk. Neurology 1999;53:251-59

8. Powers WJ. Cerebral hemodynamics in ischemic cerebrovascular disease. Ann Neurol 1991;29:231-40

9. Rapela CE, Green HA. Autoregulation of canine cerebral blood flow. Circ Res 1964;15:205-11

10. Eskey CJ, Sanelli PC. Perfusion imaging of cerebrovascular reserve. Neuroimaging Clin N Am 2005;15:367-81

11. Reinhard M, Hetzel A, Lauk M, et al. Dynamic cerebral autoregulation testing as a diagnostic tool in patients with carotid artery stenosis. Neurol Res 2001;23:55-63

12. Eicke BM, Buss E, Bahr RR, et al. Influence of acetazolamide and $\mathrm{CO} 2$ on extracranial flow volume and intracranial blood flow velocity. Stroke 1999;30:76-80

13. Schreiber SJ, Gottschalk S, Weih M, et al. Assessment of blood flow velocity and diameter of the middle cerebral artery during the acetazolamide provocation test by use of transcranial Doppler sonography and MR imaging. AJNR Am J Neuroradiol 2000;21:1207-11

14. Valdueza JM, Draganski B, Hoffmann O, et al. Analysis of CO2 vasomotor reactivity and vessel diameter changes by simultaneous venous and arterial Doppler recordings. Stroke 1999;30:81-86

15. Huber P, Handa J. Effect of contrast material, hypercapnia, hyperventilation, hypertonic glucose and papaverine on the diameter of the cerebral arteries: angiographic determination in man. Invest Radiol 1967;2:17-32

16. Schoonman GG, van der Grond J, Kortmann C, et al. Migraine headache is not associated with cerebral or meningeal vasodilatation: a 3T magnetic resonance angiography study. Brain 2008;131:2192-200

17. Bamford JM, Sandercock PA, Warlow CP, et al. Interobserver agreement for the assessment of handicap in stroke patients. Stroke 1989;20:828

18. Hoogeveen RM, Bakker CJ, Viergever MA. Limits to the accuracy of vessel diameter measurement in MR angiography. J Magn Reson Imaging 1998;8:1228-35

19. Hendrikse J, Eikelboom BC, van der Grond J. Magnetic resonance angiography of collateral compensation in asymptomatic and symptomatic internal carotid artery stenosis. J Vasc Surg 2002;36:799-805

20. Vorstrup S, Henriksen L, Paulson OB. Effect of acetazolamide on cerebral blood flow and cerebral metabolic rate for oxygen. J Clin Invest 1984;74:1634-39

21. Lassen NA, Friberg L, Kastrup J, et al. Effects of acetazolamide on cerebral blood flow and brain tissue oxygenation. Postgrad Med J 1987;63:185-87

22. Derdeyn CP, Videen TO, Yundt KD, et al. Variability of cerebral blood volume and oxygen extraction: stages of cerebral haemodynamic impairment revisited. Brain 2002;125(pt 3):595-607

23. Ozgur HT, Kent WT, Masaryk A, et al. Correlation of cerebrovascular reserve as measured by acetazolamide-challenged SPECT with angiographic flow patterns and intra- or extracranial arterial stenosis. AJNR Am J Neuroradiol 2001;22:928-36

24. Chen A, Shyr MH, Chen TY, et al. Dynamic CT perfusion imaging with acetazolamide challenge for evaluation of patients with unilateral cerebrovascular steno-occlusive disease. AJNR Am J Neuroradiol 2006;27:1876-81

25. Kikuchi K, Murase K, Miki H, et al. Measurement of cerebral hemodynamics with perfusion-weighted MR imaging: comparison with pre- and post-acetazolamide 133Xe-SPECT in occlusive carotid disease. AJNR Am J Neuroradiol 2001;22:248-54

26. Detre JA, Samuels OB, Alsop DC, et al. Noninvasive magnetic resonance imaging evaluation of cerebral blood flow with acetazolamide challenge in patients with cerebrovascular stenosis. J Magn Reson Imaging 1999;10:870-75

27. Orosz L, Fulesdi B, Hoksbergen A, et al. Assessment of cerebrovascular reserve capacity in asymptomatic and symptomatic hemodynamically significant carotid stenoses and occlusions. Surg Neurol 2002;57:333-39 